\title{
Magnetic resonance in high-temperature superconductors
}

\author{
K N SHRIVASTAVA
}

School of Physics, University of Hyderabad, P.O. Central University, Hyderabad 500134 , India

\begin{abstract}
Since the discovery of high-temperature superconductors a new microwave absorption has been found. The microwaves are absorbed in the flux-quantized eigenstates so that the absorption is proportional to the Josephson current and hence it varies as the gap of the superconductor. This absorption is found in the electron-paramagnetic resonance configuration. The flux-quantized fields are found in small domains of the size of $10^{-6} \mathrm{~cm}$. A giant moment is found to occur. The necessary theory as well as experiments in $\mathrm{YBa}_{2} \mathrm{Cu}_{3} \mathrm{O}_{7-\delta}$ type compounds are described. The $\mathrm{Cu}^{2+}$ electron-paramagnetic resonance gives an anisotropic exchange narrowed line with anisotropic $g$-values with $g$-shift proportional to susceptibility. The symmetry of the $g$-value also reflects the symmetry of the superconducting gap.
\end{abstract}

Keywords. Magnetic resonance; microwave absorption; low-field microwave signals; giant moments; EPR.

\section{Introduction}

Since the discovery of high-temperature superconductors we have reported the electron-paramagnetic resonance spectra and a new microwave absorption (Shrivastava 1987, 1988a, b, c). It is also found that pairs of electrons may be formed in the valence band as well as in the conduction band so that the range of energy in which the electrons are attractive, is larger in the present theory (Shrivastava 1989) than in the BCS theory.

In this paper, we report the theory of microwave absorption and magnetic resonance in some high-temperature superconductors along with some experimental data.

\section{Low-field microwave signals}

In all the samples, there is a strong microwave absorption at very low fields. The intensity of this line vanishes in the paramagnetic phase above the superconducting transition temperature and the line shows hysteresis. We also found that the intensity of the microwave signal is proportional to the surface area. As the system is cooled there is a shift towards higher fields.

The low-field peak corresponds to the maximum value of the Josephson current and the intensity is related to the gap of the superconductor. The Josephson current is given by

$$
J=J_{0} \sin \left(\gamma_{1}-\gamma_{2}-\frac{2 e}{h} \int \mathbf{A} \cdot \mathrm{dl}\right)
$$

where $\gamma_{1}$ and $\gamma_{2}$ are phase factors, $\mathbf{A}$ is the vector potential of the electromagnetic wave and the integral is extended over the width of the Josephson junction. Since

$$
J_{0}=\pi \Delta / 2 S R_{N}
$$


with $\Delta$ the gap of the superconductor, $R_{N}$ the normal state resistivity of the insulating region and $\delta$ the surface area, the intensity of the signal of the microwave absorption varies as the gap, $\Delta$, of the superconductor. The zero of the $\Delta$ determines the transition temperature, $T_{c}$. In the case of type-II superconductors, the Abrikosov vortex state has two critical fields, $H_{c 1}=\phi_{0} / \pi \lambda^{2}$ and $H_{c 2}=\phi_{0} / \pi \xi^{2}$ which are related to two characteristic distances in the superconductor, the London penetration depth $\lambda$ and the coherence length $\xi$. The area in (2) is independent of the critical fields, $H_{c 1}$ or $H_{c 2}$ as the zeros of (1) are not critical. Finite current occurs as the phase moves away from the zero of (1) by arbitrarily small amounts.

In the electron-paramagnetic resonance spectrum of a small sample of $\mathrm{ErBa}_{2} \mathrm{Cu}_{3} \mathrm{O}_{7-\delta}(\delta \simeq 0 \cdot 2)$, we find a large number of equally-spaced lines at $9 \cdot 2 \mathrm{GHz}$ at $4.2 \mathrm{~K}$ as shown in figure 1 . These lines are centred at about $3.8 \mathrm{G}$ and the line spacing is about $0.16 \mathrm{G}$ at the microwave power of $0.03 \mathrm{~mW}$. As the power is increased, the lines saturate as normally expected. Upon further increase of power of about $1 \mathrm{~mW}$, every line further splits and new lines arise within each line. This time the line spacings are about $0.2 \mathrm{G}$ only. We attempt to understand these line spacings in terms of vortex sizes. The large spacing of $0 \cdot 16 \mathrm{G}$ then corresponds to a small Josephson vortex of size $\lambda_{J} \simeq\left(\phi_{0} / \delta H\right)^{1 / 2} \simeq 11.37 \times 10^{-4} \mathrm{~cm}$ which upon increase of the microwave power becomes a large continuous vortex of size $\lambda$ and hence smaller line spacings $\delta H=0.02 \mathrm{G}, \lambda \simeq 32.15 \times 10^{-4} \mathrm{~cm}$. The centre of the spectrum at $3.8 \mathrm{G}$ corresponds to the largest of the fields and hence to the smallest of the dimensions compared with $\lambda_{J}$ or $\lambda$, we call it the core size, $\lambda_{c}$. Using the relation $h v=g_{s} \mu_{s} H_{0}$, we find for $H_{0}=3.8 \mathrm{G}$ and $v=9.2 \mathrm{GHz}, g_{\mathrm{s}} \mu_{s}=16.04 \times 10^{-18} \mathrm{erg} / \mathrm{G}$ which compared with Bohr magneton $\mu_{B}$ is equal to $g_{s} \mu_{s}=865 g_{e} \mu_{B}$. This is the giant magnetic moment. If we put $g_{s}=g_{e}$, then $\mu_{s}=865 \mu_{B}$. If $\mu_{s}$ is composed of many occupied sites of electrons,
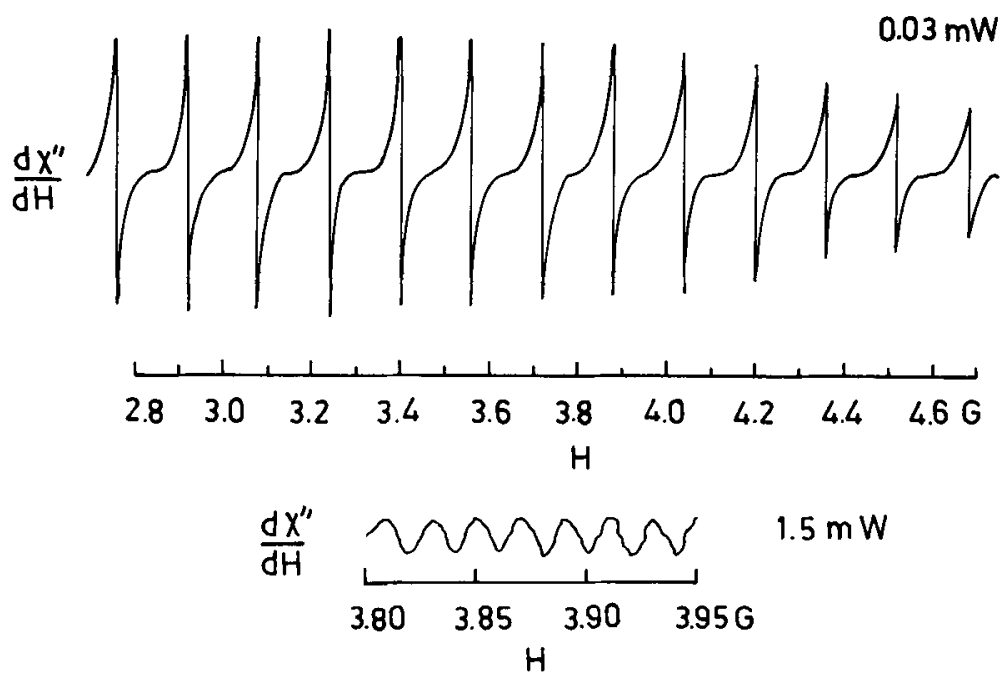

Figure 1. Low field microwave absorption in a small, $-0.01 \mathrm{~cm}$ size single crystal of $\mathrm{ErBa}_{2} \mathrm{Cu}_{3} \mathrm{O}_{7}$ at $9 \cdot 2 \mathrm{GHz}$ frequency for the magnetic field along [110] direction. The upper spectrum is taken for $0.03 \mathrm{~mW}$ power. Then the shape of one of the lines is recorded with increasing microwave power till it saturates. Upon further increase of microwave power, the lines split into several lines as shown in the lower spectrum at $1.5 \mathrm{~mW}$. 
this means that 865 sites are needed to explain $\mu_{s}$. Assuming that these sites are in a square plane, the number of sites in a line would amount to 29 . Since the length of the unit cell is about $3.8 \AA$, this amounts to a distance of $0.01 \times 10^{-4} \mathrm{~cm}$. This distance may be taken as a measure of the size of core, $\lambda_{c}$. Thus we have $\lambda_{c} \ll \lambda_{J}<\lambda$. The vortex structure in the presence of a radio frequency field has been described by Thiemann et al (1989). The importance of various lengths such as $\lambda_{J}$ and $\lambda$ in relation to the grain size $d$ is given by Kohl et al (1989) and by Sonin (1988) and the possibility of a large $g$-value $\sim 200$, has been discussed by Vesnin et al (1988). The microwave absorption has also been discussed in many other papers such as those of Portis et al (1988), Ciccarello et al (1989), Mehran et al (1988) and Misra and Misiak $(1989 a, b)$.

\section{Giant moments}

We find that fine equally spaced lines arise when a small $\left(\sim 5 \times 10^{-6} \mathrm{~cm}^{3}\right)$ single crystal of $\mathrm{ErBa}_{2} \mathrm{Cu}_{3} \mathrm{O}_{7-\delta}(\delta \simeq 0 \cdot 2)$ is placed in the electron-paramagnetic resonance spectrometer operating at $x$-band frequencies with the magnetic field parallel to the crystallographic $c$-axis at $\sim 4.2 \mathrm{~K}$. The line spacing $\delta H=0.116 \mathrm{G}$ at the microwave frequency, $v=9.4 \mathrm{GHz}$, is minimum for the field along [110] direction and the centre field is $H_{0}=3.8 \mathrm{G}$. All the equally spaced lines can be described by

$$
H_{n}=H_{0} \pm \delta H\left(n+\frac{1}{2}\right)
$$

where $n$ is an integer $(n=0,1,2 \ldots)$. Here the positive sign gives the lines on the right hand side of the centre and the negative sign gives those on the left. We look for a hamiltonian to describe the pattern of lines in the spectra. The major terms are of the form,

$$
H=g_{s} \mu_{s} H S_{z}+\sum_{i} h \omega_{i} a_{i}^{\dagger} a_{i}+g_{s} \mu_{s} \delta H_{z} \sigma_{z} S_{z}\left(a_{i}+a_{-i}^{\dagger}\right)\left(a_{j}+a_{-j}^{\dagger}\right),
$$

where $g_{s} \mu_{s}$ is the effective magnetic moment of the object in the pattern, $S_{z}$ is the $z$-component of its spin and $\sigma_{z}$ one more spin component. For $S=\sigma=\frac{1}{2}$, the above has the eigenvalues, $E_{s_{x}, \sigma_{z}, n}$ given as follows:

$$
\begin{aligned}
& E_{++n}=\frac{1}{2} g_{s} \mu_{s} H+n \hbar \omega+\frac{1}{2} g_{s} \mu_{s} \delta H\left(n+\frac{1}{2}\right), \\
& E_{+-n}=\frac{1}{2} g_{s} \mu_{s} H+n \hbar \omega-\frac{1}{2} g_{s} \mu_{s} \delta H\left(n+\frac{1}{2}\right), \\
& E_{-+n}=-\frac{1}{2} g_{s} \mu_{s} H+n \hbar \omega+\frac{1}{2} g_{s} \mu_{s} \delta H\left(n+\frac{1}{2}\right), \\
& E_{--n}=-\frac{1}{2} g_{s} \mu_{s} H+n \hbar \omega-\frac{1}{2} g_{s} \mu_{s} \delta H\left(n+\frac{1}{2}\right),
\end{aligned}
$$

where we used the exact correlation function $\left(a_{i}+a_{-i}^{\dagger}\right)\left(a_{j}+a_{-j}^{\dagger}\right)=(2 n+1) \delta_{i j}$ with $n$ as the Bose number density, $n=\left\langle a_{i}^{\dagger} a_{i}\right\rangle$. Taking the energy differences

$$
\begin{aligned}
& E_{++n}-E_{--n}=g_{s} \mu_{s} H+g_{s} \mu_{s} \delta H\left(n+\frac{1}{2}\right), \\
& E_{+-n}-E_{-+n}=g_{s} \mu_{s} H-g_{s} \mu_{s} \delta H\left(n+\frac{1}{2}\right),
\end{aligned}
$$

with $n=0,1,2, \ldots$ in these two relations, we find the energies $g_{s} \mu_{s}\left(H \pm \frac{1}{2} \delta H\right), g_{s} \mu_{s}$ $\left(H \pm \frac{3}{2} \delta H\right), g_{s} \mu_{s}\left(H \pm \frac{5}{2} \delta H\right), \ldots$ where the first relation in (6) has the positive sign and hence describes the spectral lines on the right hand side of the centre, while the second relation has negative sign and hence lines on the left hand side. 


\section{Electron-paramagnetic resonance}

$\mathrm{Cu}^{2+}$ gives the electron-paramagnetic resonance (EPR) signals with $g_{\|}=2 \cdot 216 \pm 0 \cdot 001$ and $g_{\perp}=2.047 \pm 0.001$. Near the transition temperature $g_{\|}$shows a mean field exponent. Below $T_{c}$ the $g$-value reduces in magnitude. The quantity $g_{\perp}(R T)-g_{\perp}$ increases from 0 to about 0.005 in going from $90 \mathrm{~K}$ to $79 \mathrm{~K}$. This means that the $g$-value reduces by about 0.005 per Kelvin. This reduction is caused by a contribution to the $g$-value proportional to the susceptibility. As the sample is cooled below $T_{c}$, it becomes superconducting so that the susceptibility reduces owing to negative diamagnetic contribution leading to the reduced $g$-value. The $g_{\|}$is comparatively less sensitive to this phase change. As the $\mathrm{Cu}^{2+}-\mathrm{O}^{2-}$ ions form planes we expect the $d$-orbitals to be more susceptible to the changes in the $a b$-plane than in the $c$-direction. The $g$-shift, $\delta g \sim \chi$, being proportional to the susceptibility reflects the symmetry of the superconducting state. In the $a b$-plane, below $T_{c}$, the susceptibility becomes negative so that $g_{\perp}$ reduces, while $g_{\|}$remains nearly constant. This means that the $d$-orbital is aligned along the $c$-direction and the superconducting electrons are mostly in the $a b$-plane.

\section{Non-observation of EPR}

The EPR signals may not be observed due to the metallic nature of the samples as the r.f. field does not penetrate the surface. In such a case, it is necessary to make a powder of the sample which can be suspended in an oil to allow for the penetration of the microwave field. If the signals are visible in the normal state which disappear in the superconducting state, then such an effect may be due to broadening of the lines. Mehran and Anderson (1989) suggest that a broadening of the form

$$
\Delta H=\Delta H_{N}(\xi / a)
$$

may be caused by the coherence. Here $\Delta H_{N}$ is the line width in the normal state, $\xi$ is the coherence length and $a$ is the interatomic distance within the crystallographic unit cell. In another work (Shrivastava 1990) we find that the width may be given by flux quantization as

$$
\Delta H=\Delta H_{N}\left(\phi_{0} / B\right)^{1 / 2}(1 / a)
$$

\section{Conclusions}

For several years, we have been studying (Shrivastava 1975) the interaction of paramagnetic electrons with lattice vibrations which we reviewed in 1975. In particular, it was found that there are oscillations in solids which can be seen by the shift of the paramagnetic resonance lines as a function of temperature (Shrivastava 1969, 1988d) and the shift changes sign (Singh and Shrivastava 1980; Shrivastava 1984) thus indicating the possible attractivity between electrons. In the case of $\mathrm{Cr}^{3+}: \mathrm{BaTiO}_{3}$ our shift was measured by Muller et al (1985). This work is thus at the outset of the discovery.

In the high-temperature superconductors, the type-II character is quite clear which explains the low-field microwave absorption in terms of flux-quantized eigenvalues. 
The magnetic resonance also measures the symmetry of the superconducting state. In some cases the relaxation time measurements lead to a determination of the gap energy of the superconductor.

\section{References}

Ciccarello I, Guccione M and Vigni M Li 1989 Physica C161 39

Kohl M, Odehnal M, Plintovic M and Safrata S 1989 J. Low Temp. Phys. 74503

Mehran F and Anderson P W 1989 Solid State Commun. 7129

Mehran F, Barnes S E, Giess E A and McGuire T R 1988 Solid State Commun. 6755

Misra S K and Misiak L E 1989a Solid State Commun. 72117

Misra S K and Misiak L E 1989b Solid State Commun. 72351

Muller K A, Berlinger W and Albers J 1985 Phys. Rev. B32 5837

Portis A M, Blazey K W, Muller K A and Bednorz J G 1988 Europhys. Lett. 5467

Shrivastava K N 1969 Phys. Rev. 187446

Shrivastava K N 1975 Phys. Rep. C20 137

Shrivastava K N 1984 in XXII Congress Ampere, Zurich, (eds) K A Muller, R Kind and J Roos, (Switzerland: University of Zurich Press) p. 239

Shrivastava K N 1987 J. Phys. C20 L789

Shrivastava K N 1988a Solid State Commun. 68259

Shrivastava K N 1988b Solid State Commun. 681019

Shrivastava K N 1988c J. Phys. (Paris) 49 C8-2239

Shrivastava K N 1988d Proc. R. Soc. (London) A419 287

Shrivastava K N 1989 Solid State Commun. 691173

Shrivastava K N 1990 (unpublished results)

Singh A and Shrivastava K N 1980 Phys. Status Solidi B101 51

Sonin E B 1988 JETP Lett. 47496

Thiemann S L, Radovic Z and Kogan V G 1989 Phys. Rev. B39 11406

Vesnin Yu I, Istomin V E and Kostsov E G 1988 JETP Lett. 48374 